\title{
Monitoreo DE CONCENTRACIÓN DE MONÓXIDO DE CARBONO USANDO TECNOLOGÍA LONG-RANGE
}

\section{Carbon Monoxide Concentration MONITORING USING LONG-RANG TECHNOLOGY}

\author{
José Ignacio Vega-Luna ${ }^{1, *}$, Mario Alberto Lagos-Acosta ${ }^{1}$, Gerardo Salgado-Guzmán $^{1}$
}

\section{Resumen}

Se presenta un sistema de monitoreo remoto de sensores de monóxido de carbono ( $\mathrm{CO}$ ) en un hospital usando transceptores Long-Range. Un gas altamente tóxico que no puede ser detectado por el ser humano y que causa daños en la salud es el CO. Existen zonas de hospitales donde la pureza del aire es mayor a $90 \%$ y es monitoreada continuamente. El objetivo de este trabajo fue diseñar un sistema inalámbrico que reporte a un servidor ubicado en la Internet los niveles de concentración de $\mathrm{CO}$ de diez sensores dispuestos en áreas de un hospital. Se implantó una red de área amplia de bajo consumo de energía, o LPWAN por sus siglas en inglés, utilizando diez transceptores de tecnología Long-Range y un gateway. Cada nodo de la LPWAN consta de un sensor de CO, un módulo de sistema de posicionamiento global, un display de cristal líquido de cuarzo, un generador de alerta, un microcontrolador y un transceptor Long-Range. Los nodos transmiten los niveles de $\mathrm{CO}$ al gateway y este los envía al servidor. $\mathrm{Al}$ detectarse un nivel de $\mathrm{CO}$ mayor a un valor de umbral se activa una alerta. El sistema detecta concentraciones de CO de 10 a 1000 ppm. El nodo de la red más lejano se encuentra a 1200 metros del gateway y el alcance logrado fue 11,8 kilómetros.

Palabras clave: hospital, Long-Range, LPWAN, monóxido de carbono sensor, transceptor.

\section{Abstract}

A remote monitoring system for carbon monoxide (CO) sensors is presented in a hospital using LongRange transceivers. A highly toxic gas that can't be detected by humans and damages health is CO. There are hospital areas where air purity is greater than $90 \%$ and monitored continuously. The objective of this work was to design a wireless system that reports to a server, located on the Internet, the concentration levels of $\mathrm{CO}$ around ten sensors distributed around the areas of a hospital. A low-power wide area network (LPWAN) was deployed using ten Long-Range technology transceivers and a gateway. Each node of the LPWAN consists of a CO sensor, a global positioning system module, a quartz liquid crystal display, an alert generator, a microcontroller and a Long-Range transceiver. The nodes transmit the $\mathrm{CO}$ levels to the gateway and the gateway sends them to the server. When a CO level higher than a threshold value is detected, an alert is triggered. The system detects CO concentrations of 10 to $1,000 \mathrm{ppm}$. The farthest network node is located 1,200 meters from the gateway and the range achieved was 11.8 Kilometers.

Keywords: Carbon monoxide, hospital, Long-Range, LPWAN, sensor, transceiver.

\footnotetext{
$1, *$ Área de Sistemas Digitales, Departamento de Electrónica, Universidad Autónoma Metropolitana-Azcapotzalco, Cd. de México, México. Autor para correspondencia: vlji@correo.azc.uam.mx, (1) http://orcid.org/0000-0002-4226-2936, (D) http://orcid.org/0000-0003-0455-007X, (D) http://orcid.org/0000-0002-0581-7410
} 


\section{Introducción}

En hospitales, laboratorios clínicos y centros de salud es importante mantener el aire libre de impurezas, bacterias y gases tóxicos. Uno de estos gases es el monóxido de carbono ( $\mathrm{CO}$ ) [1]. En zonas de hospitales, como quirófanos, tratamiento de enfermedades de piel y pulmones, así como anestesiología, la pureza del aire debe ser mayor a $90 \%$ [2-4]. El monóxido de carbono es un gas inodoro, incoloro, insípido que no puede ser detectado por los sentidos humanos. En ocasiones se le llama el asesino silencioso, ya que solo puede ser descubierto con un dispositivo especial. El CO se genera por mala combustión de materiales fósiles en cocinas, hornos, calentadores, chimeneas, automóviles y calderas [5].

Cuando una persona respira aire contaminado con monóxido de carbono, las moléculas de $\mathrm{CO}$ entran a los pulmones y se transfieren al sistema sanguíneo. Esto provoca falta de oxígeno en el cuerpo, incluyendo el cerebro. En una persona expuesta a bajas concentraciones de $\mathrm{CO}$, se acumula gradualmente en la sangre, provoca dolor de cabeza, náuseas y fatiga. Si la persona no advierte que lo está respirando, entrará en estado de somnolencia hasta dormirse. Si las concentraciones de CO son altas, provocará en la persona confusión, pérdida de conocimiento, problemas cardíacos y pulmonares y, en el peor de los casos, la muerte [6]. Por tales razones, el $\mathrm{CO}$ se clasifica como gas altamente tóxico y puede estar presente en cualquier lugar. Autoridades sanitarias y de protección civil de algunas ciudades exigen la instalación de detectores de humo y CO, no solo en hospitales, sino también en hogares, oficinas, industrias e instalaciones educativas. La concentración de $\mathrm{CO}$ en el aire del ambiente se mide en partes por millón (ppm). Cuando una persona está expuesta a CO puede presentar diversos efectos dependiendo de la concentración del gas, como se indica en la Tabla 1.

El objetivo de este trabajo fue implantar un sistema que registre periódicamente en un servidor ubicado en la Internet el valor de concentración de CO en el ambiente de distintos puntos de un hospital.

Existen diferentes tipos de sensores para medir la concentración de $\mathrm{CO}$ en el aire [7], los más comúnmente usados en soluciones comercialmente disponibles son los que se explican a continuación:

Sensores electroquímicos. Son sensores de estado sólido que tienen una barrera de difusión capilar que controla la entrada del $\mathrm{CO}$ al interior de una cápsula. En la cápsula se encuentran dos electrodos y un electrolito, generalmente ácido sulfúrico. Al pasar el $\mathrm{CO}$ a la cápsula, un electrodo se oxida y el otro consume oxígeno. Se genera una corriente eléctrica cuyo valor es proporcional a la concentración de gas. Cuando el nivel de $\mathrm{CO}$ alcanza un valor de umbral se activa una alarma. Son sensores muy precisos y en ocasiones forman parte de un sistema de detección que incluye un dispositivo para mostrar el valor de concentración de CO y una memoria para almacenar históricamente los valores registrados. Es el tipo de sensor más usado porque trabaja libre de mantenimiento durante periodos prolongados de tiempo y es bastante estable y confiable.

Sensores biométricos, infrarrojos o catalíticos. Usan una solución líquida similar a la sangre. La solución está compuesta por una mezcla de sales de metales, azúcar y una sustancia química. El sensor tiene un emisor de luz infrarroja cuyo haz pasa por la solución líquida y es recibida por un fotodiodo. Cuando detecta una concentración peligrosa de $\mathrm{CO}$, el color de la solución se torna oscuro y se interrumpe el haz de luz infrarroja que activa una alarma. Estos sensores se usan para detectar un nivel de concentración de CO de umbral y no realizan mediciones continuas.

Sensores de semiconductor. Utilizan una fuente de corriente controlada por un circuito integrado. La corriente circula por cables de dióxido de estaño que al detectar un nivel de concentración alto de CO se cierra el circuito eléctrico y se activa una alarma. Consumen más energía eléctrica que los anteriores y deben conectarse a la instalación eléctrica del lugar donde se usan. Algunos modelos incluyen una batería de respaldo.

Tabla 1. Concentraciones de $\mathrm{CO}$ y efecto en el ser humano

\begin{tabular}{|c|c|}
\hline $\begin{array}{l}\text { Concentración } \\
\text { de CO (ppm) }\end{array}$ & Efecto \\
\hline 50 & $\begin{array}{l}\text { Nivel de exposición admisible } \\
\text { durante } 8 \text { horas. }\end{array}$ \\
\hline 200 & $\begin{array}{l}\text { Dolor de cabeza moderado } \\
\text { después de } 2 \text { horas. }\end{array}$ \\
\hline 400 & $\begin{array}{l}\text { Dolor de cabeza y náuseas des- } \\
\text { pués de } 1 \text { o } 2 \text { horas. }\end{array}$ \\
\hline 800 & $\begin{array}{l}\text { Dolor de cabeza, mareos y } \\
\text { náuseas después de } 45 \text { minu- } \\
\text { tos. Descompensación y riesgo } \\
\text { de muerte después de } 2 \text { horas. }\end{array}$ \\
\hline 1600 & $\begin{array}{l}\text { Dolor de cabeza, mareos y } \\
\text { náuseas después de } 20 \text { minu- } \\
\text { tos. Descompensación y riesgo } \\
\text { de muerte después de } 1 \text { hora. }\end{array}$ \\
\hline 3200 & $\begin{array}{l}\text { Dolor de cabeza y mareos des- } \\
\text { pués de } 5 \text { a } 10 \text { minutos. Pér- } \\
\text { dida de conciencia y riesgo de } \\
\text { muerte después de } 30 \text { minutos. }\end{array}$ \\
\hline 6400 & $\begin{array}{l}\text { Dolor de cabeza y mareos des- } \\
\text { pués de } 1 \text { a } 2 \text { minutos. Pér- } \\
\text { dida de conciencia y riesgo de } \\
\text { muerte después de } 10 \text { a } 15 \text { mi- } \\
\text { nutos. }\end{array}$ \\
\hline 12800 & $\begin{array}{l}\text { Efectos inmediatos, pérdida } \\
\text { de conciencia. Riesgo de } \\
\text { muerte después de } 1 \text { a } 3 \text { minu- } \\
\text { tos. }\end{array}$ \\
\hline
\end{tabular}


Se han realizado sistemas de detección de CO que usan sensores de tecnología más reciente, ultrasensibles y experimentales, aún no disponibles comercialmente. Algunos de estos sensores están basados en nano películas bimetálicas [8] o semiconductoras de teluro de cadmio $(\mathrm{CdTe})$ [9]. Otros sistemas utilizan un detector infrarrojo no dispersivo o NDIR (por sus siglas en inglés), los cuales determinan la concentración de CO midiendo la absorción de la longitud de onda del gas en el infrarrojo [10]. Se han desarrollado sensores de CO con láseres en cascada de cavidad continua ( $C W, D F B$ QCL-Continuous Wave, Distributed-FeedBack Quantum Cascade Laser), los cuales requieren una fuente de alto poder [11].

También se han creado aplicaciones que miden la concentración de $\mathrm{CO}$ en distintos ambientes y con objetivos diferentes al planteado en este trabajo. Por ejemplo se han realizado implantaciones para monitorear la calidad del aire en minas de carbón [12] y en la construcción de obras subterráneas para evitar el envenenamiento de trabajadores [13]. Otros trabajos determinan el grado de contaminación de la atmósfera y patrones del clima analizando la concentración de $\mathrm{CO}$ en aire mediante información obtenida por satélites [14]- [15]. En el sector de la salud también se han realizado trabajos para detectar clínicamente la saturación de la sangre y envenenamiento por monóxido de carbón en personas fumadoras [16].

Algunos sistemas de monitoreo de CO actualmente disponibles solo muestran y registran localmente el nivel de CO detectado y activan una alarma en caso de que el nivel alcance un valor de umbral. Los que usan tecnología más reciente están basados en una red de sensores que transmiten a un equipo central los niveles de $\mathrm{CO}$ detectados. Cuando la distancia entre el sensor y el punto de monitoreo es menor a 50 metros, comúnmente se usa cableado directo o transmisores de radio de corto alcance, máximo 40 metros. Para distancias mayores a 50 metros, la comunicación se realiza usando transceptores de tecnología inalámbrica tradicional como wifi, ZigBee o Bluetooth $[17,18]$.

En los últimos años se han realizado trabajos donde los sensores se conectan a un transceptor para transmitir a una estación central, conectada a la Internet, el nivel de concentración de $\mathrm{CO}$ a través de un ruteador [19]. Las desventajas de estas redes de sensores son: el alcance en decenas de metros, el alto consumo de energía y el alto costo de la infraestructura. Se han desarrollado bastantes implantaciones donde los sensores de $\mathrm{CO}$ se conectan a una red de transceptores ZigBee, los cuales consumen mucho menos energía que wifi, pero su alcance está limitado a 100 metros [20].

Si la estación central está ubicada en la Internet, debe usarse un gateway ZigBee [21]. ZigBee no puede comunicarse fácilmente con otros protocolos [22]. La red de sensores con tecnología wifi tiene mayor ancho de banda que la red ZigBee, sin embargo, el costo de transceptores y gateways wifi es más elevado que ZigBee, consumen mayor cantidad de energía y el alcance de wifi está reducido a decenas de metros. Las redes wifi se usan cuando se transmiten grandes cantidades de información. En una red de sensores la cantidad de información transmitida es pequeña y no se transmite continuamente [23].

También se ha usado tecnología Bluetooth para la transmisión de información, adquirida por sensores de CO, a teléfonos móviles o a una computadora [24], lo cual presenta la desventaja de tener alcance limitado de metros por lo que se usa comúnmente para la conexión de dispositivos personales. Adicionalmente, se han realizado trabajos de investigación que miden la calidad del ambiente y concentración de CO, transmitiendo la información de sensores usando tecnología celular GSM [25]. Su alcance es ilimitado, pero tienen la desventaja del costo de uso de la red celular.

En cualquiera de las tecnologías inalámbricas anteriores debe instalarse y configurarse la computadora que realiza la tarea de estación central y registra la información recabada por los sensores o el teléfono móvil y la aplicación correspondiente en el caso de Bluetooth. Recientemente ha surgido la tecnología Bluetooth Smart o Bluetooth Low Energy (BLE). Es de bajo consumo de energía y está dirigida con implementaciones de redes de área personal inalámbricas o WPAN (por sus siglas en inglés), con teléfonos inteligentes. Su alcance es cientos de metros, razón por la cual no se utilizó en la realización del trabajo aquí presentado.

Considerando las características de las tecnologías anteriores, la opción práctica y viable para lograr el objetivo planteado en este trabajo fue usar tecnología LoRa, desarrollando una red LPWAN de sensores de CO conectada a la Internet.

El protocolo abierto LoRa fue desarrollado por LoRa Alliance para crear redes LPWAN para el mercado de Internet de las cosas, o IoT por sus siglas en inglés [26]. LoRa define la capa física del modelo OSI, o modulación inalámbrica, para realizar el enlace de comunicación de larga distancia con transceptores de radio de baja potencia que permiten transmitir pequeñas cantidades de información a baja velocidad y lograr mayor tiempo de vida de baterías. La modulación de LoRa usa la técnica de espectro ensanchado, o CSS por sus siglas en inglés, para codificar múltiples bits por símbolo para empaquetado y corrección errores. Las LPWAN que usan el protocolo LoRa se les conoce como LoRaWAN y se usan en aplicaciones máquina a máquina o M2M, y por operadores de redes inalámbricas que usan el espectro sin licencia para comunicar dispositivos IoT a través de su red. Las LoRaWAN proporcionan mayor cobertura que las redes inalámbricas celulares existentes [27].

Muchos operadores de redes celulares complementan su oferta de redes inalámbricas/celulares con Lo- 
RaWAN ya que se integran a la infraestructura existente para ofrecer a los clientes soluciones basadas en aplicaciones de IoT alimentadas por baterías. Usando LoRaWAN pueden implantarse soluciones de IoT sin hacer uso de tecnologías de transmisión inalámbrica tradicionales. Las redes LoRaWAN usan arquitectura de estrella y los nodos establecen el enlace inalámbrico de baja frecuencia con uno o más puntos de acceso o gateways conectados a la Internet. Esta comunicación es bidireccional, segura y de baja frecuencia. Los gateways transmiten información a un servidor de red central, ubicado en la nube, usando una conexión IP estándar. El gateway es un puente transparente entre los dispositivos finales de la red y el servidor. Esto permite la interconexión entre objetos inteligentes sin necesidad de instalaciones complejas, proporcionando flexibilidad al usuario final, a desarrolladores y empresas que necesiten implantar redes de IoT. La velocidad de transmisión varía en el rango de $0.3 \mathrm{Kbps}$ a $50 \mathrm{Kbps}$ [28].

Para maximizar la duración de la batería de dispositivos finales y capacidad de la red, el servidor central LoRaWAN administra la velocidad para cada dispositivo usando un esquema adaptivo de velocidad de datos, o ADR por sus siglas en inglés. Con un gateway o estación base LoRa se pueden cubrir ciudades completas o cientos de kilómetros cuadrados. La capacidad de una LoRaWAN es de miles de nodos y usa dos capas de seguridad: una para la red y otra para la aplicación. La seguridad de la red sirve para autenticar el nodo y la seguridad en la aplicación garantiza que el operador de la red no tenga acceso a la información de la aplicación del usuario final. LoRaWAN utiliza el estándar de encriptado avanzado, o AES por sus siglas en inglés, intercambiando claves con identificadores IEEE EUI64.

Existen diferentes proveedores de transceptores y gateways LoRa para desarrollar LPWAN. Varias ciudades de Estados Unidos y Europa cuentan con operadores de LoRaWAN públicas, similares a las redes celulares existentes en casi todo el mundo, que permiten conectar una LPWAN a un servidor ubicado en la Internet y crear aplicaciones IoT. Estos operadores usan la banda ISM, que no requiere licencia, de 915 MHz. en Estados Unidos y de $868 \mathrm{MHz}$ en Europa [29]. En Latinoamérica no existe este tipo de operadores hasta hoy, por lo que una opción para conectar una LPWAN a la Internet es usando Symphony Link.

Symphony Link es una especificación alterna a LoRaWAN, es un protocolo estandarizado desarrollado por Link Labs para usuarios que necesitan comunicación inalámbrica de larga distancia con rendimiento no disponible en LoRaWAN. Symphony Link proporciona transceptores y gateways LoRa donde la comunicación es más segura que una LoRaWAN, ya que maneja reconocimiento de todos los paquetes de datos, a diferencia de una LoRaWAN que usa reconocimientos limitados presentando una tasa de error en paquetes mayor a $50 \%$ [30]. El uso de repetidores en una red permite expandir el rango de la misma aumentando también la latencia. En LoRaWAN los límites de ciclo de trabajo de la arquitectura evitan que los repetidores trabajen eficientemente. Al ser Symphony Link un protocolo síncrono, los repetidores aumentan el alcance de la red sin aumentar la latencia y su costo es mucho menor al de un punto de acceso de LoRaWAN.

Usando características síncronas, como ranuras y coordinación en los tiempos de transmisión de nodos, una red Symphony Link tiene más de cuatro veces la capacidad de una red LoRaWAN. La operación de una red Symphony Link no requiere identificador de red ni membresía a LoRa Alliance, lo cual puede costar hasta $\$ 20000$ USD por año. Symphony Link no interfiere con LoRaWAN y viceversa. Se puede desarrollar una LPWAN para IoT adquiriendo los transceptores necesarios, uno para cada nodo de la red, y el gateway Symphony Link conectado a Internet. Los nodos intercambian información, a través del gateway, con un servidor de red, ubicado en los servicios de nube de Amazon, administrado por Link Labs.

El gateway soporta más de 250000 nodos. Se han desarrollado aplicaciones usando tecnología LoRa y diferentes tipos de sensores. Por ejemplo se ha implantado una red de sensores de vibración y temperatura en un camino para analizar las condiciones del asfalto con lluvia [31]. Se ha desarrollado un sistema de navegación en el mar transmitiendo información de rutas usando transceptores LoRa [32] y se han creado sistemas con sensores de movimiento y de inclinación que permiten seguir los movimientos de una persona en el hogar o trabajo para determinar su grado de sedentarismo [33].

En este trabajo se implantó una LPWAN Symphony Link compuesta por diez nodos y un gateway. La LPWAN reporta al servidor de red periódicamente el valor de concentración de CO de cinco sensores ubicados en distintas zonas de un hospital. Cada nodo transmite al gateway el valor de concentración de CO del sensor correspondiente, juntamente con la fecha, hora y coordenadas GPS de su ubicación. Adicionalmente, los nodos cuentan con un display LCD que muestra localmente el valor de concentración de CO y una alarma audible y luminosa que se activa cuando la concentración de CO alcanza un nivel de umbral. En la interfaz de usuario, que se ejecuta en una computadora personal conectada a Internet, se muestra la información enviada por cada nodo de la red.

Las ventajas y aportaciones del sistema aquí presentado son las siguientes: la comunicación usada en la red de sensores es inalámbrica y por tanto no hubo necesidad de modificar el cableado del hospital, la tecnología inalámbrica usada es de reciente creación con alcance de varios kilómetros. No necesita instalarse una computadora para comunicarse desde la Internet a la LPWAN, ya que se usa el servidor proporcionado 
en Symphony Link. El sistema desarrollado resuelve una necesidad real, conectando una LPWAN a la Internet sin usar una LPWAN pública. Se han desarrollado diversas aplicaciones usando LoRa e IoT, pero no como la aquí realizada para hospitales.

\section{Método}

La metodología usada en el desarrollo de este trabajo consistió dividirlo en dos partes: el desarrollo de la LPWAN y la implantación de la interfaz de usuario. A continuación, se determinaron los módulos funcionales de cada una de estas partes y finalmente se construyó cada módulo usando los componentes más adecuados tomando en cuenta el consumo de energía y costo. En la Figura 1 se muestra el diagrama de bloques del sistema construido.

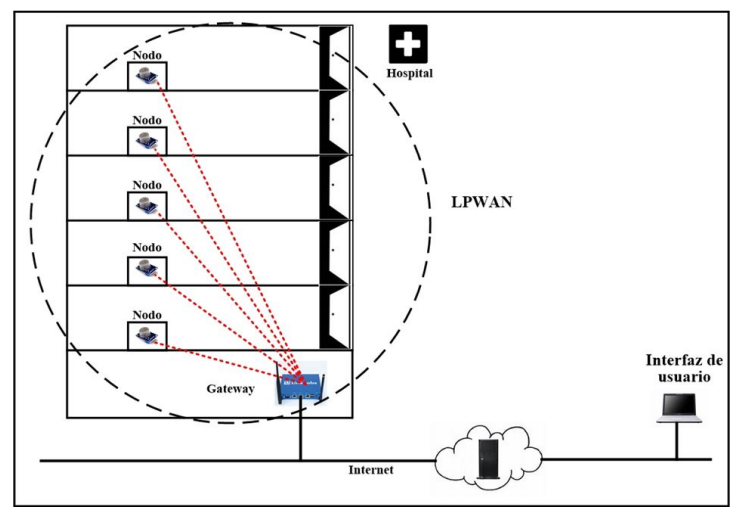

Figura 1. Diagrama de bloques del sistema.

\subsection{La LPWAN}

Se implantó una LPWAN formada por cinco nodos de la red y un gateway. Los nodos tienen la arquitectura indicada en el diagrama de bloques de la Figura 2. Cada nodo está compuesto por los siguientes elementos: el sensor de CO, el display LCD, el módulo GPS, el generador de alarma, el microcontrolador y el transceptor LoRa.

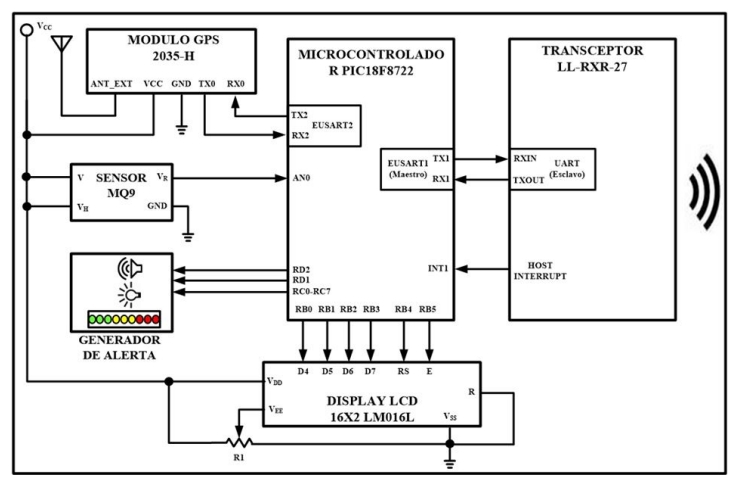

Figura 2. Diagrama de bloques de los nodos de la LPWAN.
La principal función de los nodos de la red es colectar periódicamente los valores de CO, por medio del sensor conectado al nodo, y transmitirlos al gateway. El periodo de recolección es por defecto 30 segundos y es configurable en la interfaz de usuario. El sensor de CO instalado en los diferentes pisos del hospital es el dispositivo MQ-9. Este sensor es de tipo electrolítico sólido, de bajo costo, baja dependencia de la humedad, baja conductividad en ambientes de aire limpio y detecta la presencia de monóxido de carbono, metano (CH4), propano y gas LP en el aire. Puede realizar medidas de monóxido de carbono en el rango de 10 a 1000 ppm y de metano o gas LP en el rango de 100 a 10000 ppm, siendo ideal para aplicaciones de control de pureza del aire en interiores.

Está construido con los siguientes elementos: un microtubo de cerámica $\mathrm{Al}_{2} \mathrm{O}_{3}$, una capa sensible de dióxido de estaño $\left(\mathrm{SnO}_{2}\right)$, un electrodo de medida y un calentador, todos ellos instalados en un encapsulado de plástico y una malla de acero inoxidable. El calentador genera la temperatura necesaria para el trabajo de los componentes sensibles. La conductividad del sensor aumenta conforme crece la concentración de $\mathrm{CO}$ en el ambiente. En la Figura 3 se muestra el sensor MQ-9 usado en este módulo, el cual cuenta con cuatro terminales: una se conecta al voltaje de alimentación $5 \mathrm{~V}$, otra se usa para suministrar el voltaje al calentador, la tercera terminal es tierra y la última terminal es la salida analógica $(\mathrm{AO})$.

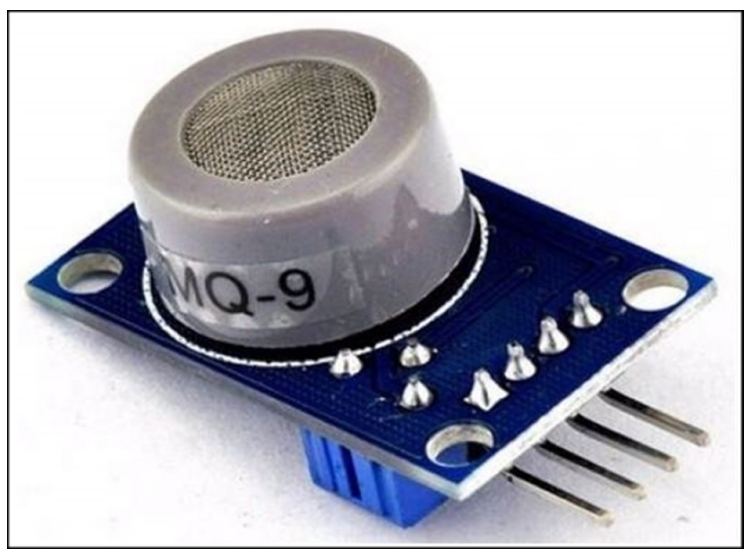

Figura 3. Sensor MQ-9.

A través de esta última terminal se obtiene el valor de concentración de CO medido por el sensor, a través de una señal de voltaje que aumenta proporcionalmente en relación con el nivel de gas detectado. El valor de esta señal es $0.1-0.3 \mathrm{~V}$ para concentraciones de $\mathrm{CO}$ menores a $10 \mathrm{ppm}$ y $4 \mathrm{~V}$ para concentraciones mayores o iguales a $1000 \mathrm{ppm}$. El MQ-9 opera a temperatura ambiente de $-10 \mathrm{a}+50{ }^{\circ} \mathrm{C}$ y consume menos de $150 \mathrm{~mA}$ a $5 \mathrm{~V}$.

En la Figura 4 se indica el circuito de prueba y calibración indicado por el fabricante del sensor. 


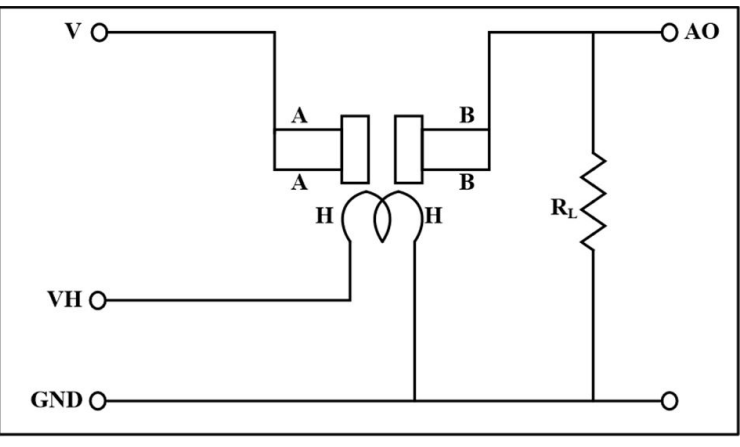

Figura 4. Circuito de prueba y calibración del sensor MQ-9.

Con este circuito se puede ajustar el valor de voltaje de la terminal de salida AO, suministrando al sensor un nivel conocido de concentración de gas y variando la resistencia de carga RL de la etapa de salida. Esta terminal se conectó a la entrada AN0 del convertidor analógico-digital (ADC) del microcontrolador.

Para determinar las coordenadas de la ubicación del sensor conectado a cada nodo de la LPWAN, se utilizó un módulo GPS A2135-H. Este módulo tiene antena de cerámica integrada y puede conectársele una antena activa externa. Contiene un motor de seguimiento de 48 canales y $-163 \mathrm{dBm}$ de sensibilidad. Su exactitud es menor a 2 metros y consume en operación $22 \mathrm{~mA}$. Transmite cada segundo las coordenadas GPS por un puerto USART, el cual conectó al EUSART2 del microcontrolador. La velocidad de este puerto serie es configurable en el rango de 4800 bps a 115,2 Kbps. La comunicación del módulo GPS con el microcontrolador se lleva cabo usando comandos que siguen el protocolo NMEA.

El microcontrolador usado en los nodos de la red es el PIC18F8722. Este dispositivo de 64 terminales se alimenta con $2 \mathrm{~V}$ a $5,5 \mathrm{~V}$ y cuenta con los siguientes recursos, suficientes para las funciones que realizan los nodos: CPU de 8 bits, $128 \mathrm{~KB}$ de memoria de programa, 3936 bytes de memoria SRAM, convertidor analógico-digital de 10 bits y 16 canales, 3 temporizadores y dos puertos EUSART. Periódicamente, el microcontrolador muestrea la entrada analógica AN0, para lo cual solicita al ADC que inicie la conversión del valor de voltaje, presente en esta entrada, a una palabra digital de 10 bits. A continuación, el PIC18F8722 espera el fin de la conversión y determina el valor de concentración de $\mathrm{CO}$ en ppm obteniendo una palabra de 16 bits.

Posteriormente, el microcontrolador lee la fecha, hora y coordenadas del módulo GPS y transmite la información del sensor y del módulo GPS, por medio del puerto EUSART1, al transceptor LoRa, para registrar y mostrar remotamente en la interfaz de usuario los niveles de concentración de CO.
El transceptor LoRa usado es el Symphony Link LL-RXR-27. Este transceptor es un radio multibanda bidireccional de Link Labs, es compatible con redes públicas LoRaWAN 1.0 y con redes privadas de área amplia Symphony Link. Está optimizado para usarse en las bandas de frecuencia de $915 \mathrm{MHz}$ ISM u $868 \mathrm{MHz}$. Usa modulación LoRa de Semtech para maximizar el alcance al mismo tiempo que minimiza el consumo de energía e interferencia. El transceptor LL-RXR-27 integra un DSP Semtech SX1276, un microcontrolador de 32 bits Renesas R5F51116ADNE. El DSP SX1276 implanta la capa física usando modulación LoRa mientras que el firmware del microcontrolador RX111 implanta la pila de protocolos de red y la interface de comandos para la comunicación con el PIC18F8722. Las características principales de operación del transceptor LL-RXR-27 son las siguientes: alimentación de 3,5 a $5,5 \mathrm{~V}$, consumo de corriente de $<1 \mathrm{uA}$ en reposo, $480 \mathrm{~mA}$ en transmisión, $40 \mathrm{~mA}$ en recepción, memoria flash de $256 \mathrm{~KB}$, memoria RAM de $32 \mathrm{~KB}$, potencia máxima de transmisión $23 \mathrm{dBm}$, velocidad de transmisión RF de 183 bps a 37,5 Kbps y velocidad de transmisión del UART de 115200 bps.

La comunicación entre el PIC18F8722 y el transceptor LL-RXR-27 se llevó a cabo conectando el puerto EUSART1 del microcontrolador al puerto UART del LL-RXR-27 para usar el protocolo maestro/esclavo. El microcontrolador realiza las funciones de maestro y el transceptor LL-RXR-27 las de esclavo. El protocolo permite intercambiar dos tipos de mensajes: paquetes de comando y paquetes de respuesta. El maestro siempre envía paquetes de comando, mientras que el esclavo siempre transmite paquetes de respuesta. Los paquetes de comando consisten de los siguientes campos: preámbulo ( 4 bytes), inicio de trama (1 byte), tipo de comando (1 byte), número de mensaje (1 byte), longitud del mensaje ( 2 bytes), mensaje (hasta 256 bytes) y checksum para verificar la integridad del paquete $(2$ bytes). Cada vez que el microcontrolador solicita al transceptor LL-RXR-27 que transmita al gateway el valor de concentración de CO y coordenadas GPS, le envía un paquete de comando indicando en el campo de mensaje estos valores. Tanto el sensor como el módulo GPS y el transceptor son dispositivos de bajo consumo de corriente que permitió crear la LPWAN con nodos de la red compactos.

El gateway usado es modelo LL-BST-8 de Link Labs, el cual, juntamente con los transceptores LLRXR-27 forman la LPWAN. La solución Symphony Link está compuesta por la LPWAN y el servidor en la nube de Link Labs. El gateway cuenta con dos puertos de red Ethernet. A través de un puerto Ethernet se conecta localmente a una computadora y por medio del otro puerto se conecta a la Internet. De esta forma, la red de sensores de $\mathrm{CO}$ puede ser accedida y monitoreada desde la Internet. Para configurar y establecer 
el hostname del gateway, el método de conexión a la Internet, ya sea alámbrico o inalámbrico, y para los parámetros de comunicación con los nodos de la red se usa la aplicación Prelude de Link Labs, la cual se ejecuta en una computadora y se comunica con el gateway a través de un segmento de red local. Cuando un transceptor LL-RXR-27 establece una sesión de comunicación con el gateway, la sesión se registra como aplicación de usuario en el gateway y en Prelude, y se le asigna un nombre o token usado para encriptar la información que transmite el gateway al servidor de red de Symphony Link.

Para mostrar localmente el nivel de concentración de $\mathrm{CO}$ presente en el ambiente, los nodos de la LPWAN cuentan con un display LCD de 4 filas de 20 caracteres cada una $(20 \mathrm{x} 4)$. El bus de datos y el bus de control del display se conectaron al puerto $\mathrm{B}$ del microcontrolador. Adicionalmente, cada nodo tiene un generador de alerta compuesto por nueve leds indicadores de concentración de $\mathrm{CO}$ dispuestos en forma de semáforo: tres leds verdes, tres amarillos y tres rojos. Cuando la concentración de CO es menor a $1000 \mathrm{ppm}$ se encienden los leds verdes, indicando calidad de aire buena. Cuando se encienden los leds amarillos la concentración de CO se encuentra entre 1000 y 2500 ppm, generándose un pequeño sonido en una bocina y cuando la concentración es mayor a $2500 \mathrm{ppm}$ se encienden los leds rojos, se activa una alerta en una bocina y se enciende una baliza estroboscópica de xenón, indicando que se debe ventilar el espacio. Los leds, la bocina y la baliza se conectaron a once líneas de los puertos C y D del microcontrolador configuradas como salidas.

La programación que se ejecuta en el microcontrolador realiza las siguientes acciones: inicializa las variables de ambiente y constantes, configura el convertidor analógico-digital, configura como salida los puertos paralelo B, C y D, configura los puertos serie EUSART1 y EUSART2, configura los temporizadores para generar la señal de reloj de los puertos serie y el periodo de muestreo de los sensores. Posteriormente entra a un ciclo donde obtiene en nivel de concentración de CO a través de la entrada AN0, determina su correspondiente valor en ppm usando una palabra de 16 bits, lee las coordenadas del módulo GPS, transmite esta información al transceptor LoRa y, finalmente, actualiza el estado del generador de alerta. En la Figura 5 se indica el diagrama de flujo de la programación que implanta las tareas anteriores.

\subsection{La interfaz de usuario}

La interfaz de usuario se realizó en Visual Basic y Python 3.6. Se ejecuta en una computadora conectada, vía la Internet, al servidor de red. El servidor de red se encuentra alojado en los servicios de nube de Amazon. La interfaz de usuario muestra el valor de concentración de CO de cada sensor en los últimos 30 segundos, como se indica en la Figura 6, y permite descargar en un archivo de texto los valores históricos registrados. El periodo de tiempo de muestreo es configurable.

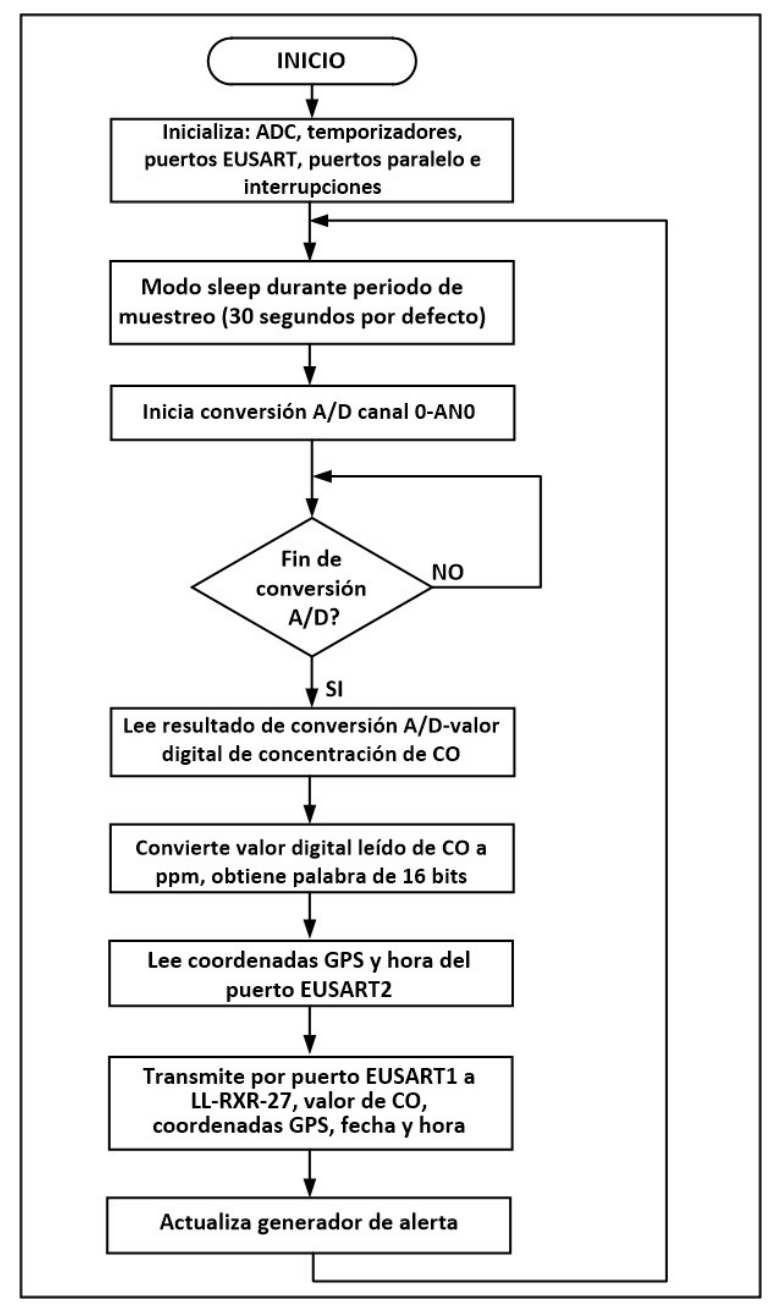

Figura 5. Interfaz de usuario.

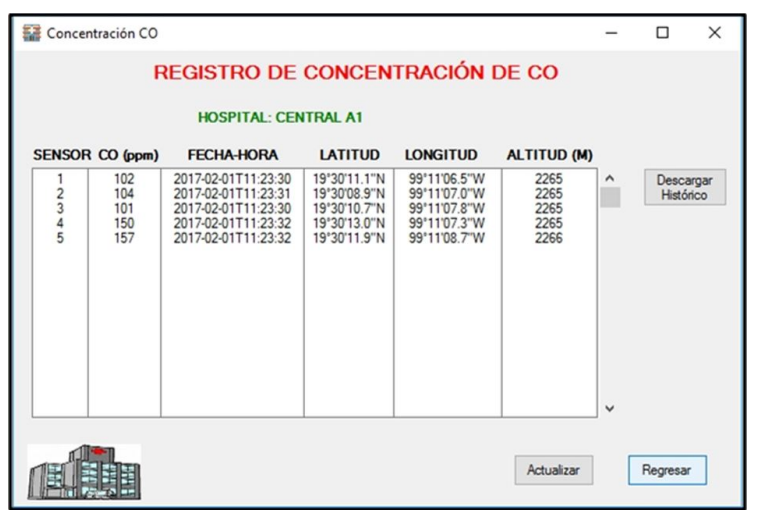

Figura 6. Diagrama de flujo de la programación del microcontrolador. 


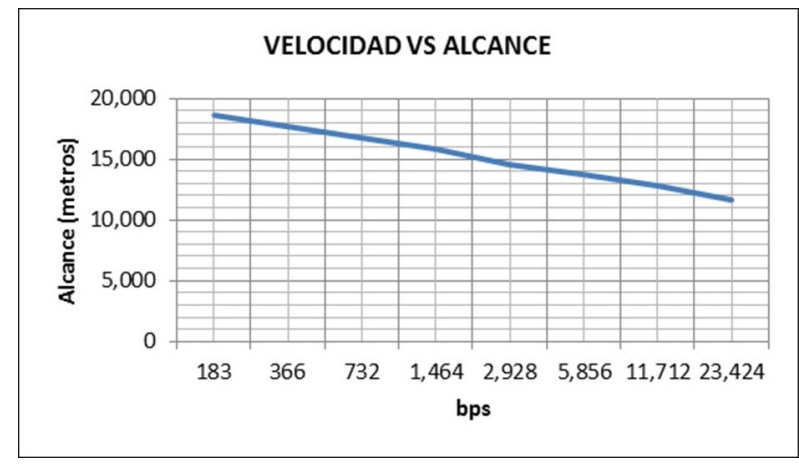

Figura 7. Alcance del transceptor LL-RXR-27.

En el servidor de red se ejecuta la aplicación Conductor de Link Labs. Esta es la plataforma de servicios de datos en la nube que puede accederse a través de un portal conectándose a la página web de Conductor. Se pueden acceder los servicios de la aplicación Conductor, desde una aplicación o programa, utilizando un conjunto de API proporcionado por Link Labs. La interfaz de usuario se conecta al portal de Conductor y usa estos API para desplegar los valores de concentración de CO y coordenadas GPS en un formato más sencillo y claro que los mostrados en el portal de Conductor. Cada vez que un gateway Symphony Link se conecta a Conductor, es registrado por esta aplicación, lo que le permite comunicarse de forma segura con cada LPWAN Symphony Link.

\section{Resultados y discusión}

El fabricante del gateway y transceptores LoRa indica que su alcance son 12 kilómetros. En este trabajo la distancia del sensor de CO más lejano al gateway es de 1200 metros y no se presentaron problemas en la comunicación en la LPWAN. Se llevaron a cabo dos grupos de pruebas con el sistema construido. El objetivo del primer grupo fue determinar el alcance de la LPWAN. Para realizar estas pruebas se ubicó un nodo de la red en distintas áreas y edificios del hospital. El alcance logrado fue 11,8 kilómetros con línea de vista, 9,1 kilómetros localizando el nodo en una posición donde existen tres edificios entre el nodo y el centro de datos y 8,2 kilómetros con cuatro edificios. El objetivo del segundo grupo de pruebas fue determinar el alcance de la LPWAN a diferentes velocidades de transmisión. En la LPWAN construida la velocidad de transmisión la establece automáticamente el gateway en el rango de 183 bps a $37.5 \mathrm{Kbps}$, dependiendo de la carga de datos que maneja en ese momento. Para realizar estas pruebas se modificó la velocidad de transmisión entre un nodo y el gateway usando la aplicación Prelude. Los resultados mostraron que a menor velocidad de transmisión, los transceptores LL-RXR-27 tienen mayor alcance. Al configurar la velocidad más baja de 183 bps se tuvo un alcance de 18,6 kilómetros con línea de vista, mayor al logrado en el primer grupo de pruebas, como se muestra en la gráfica de la Figura 7.

No es común modificar esta velocidad, Link Labs recomienda que el gateway administre los parámetros de comunicación con los transceptores, sin embargo, se realizó esta prueba para verificar cómo influye la velocidad usada en la capa física LoRa en el alcance de la LPWAN.

\section{Conclusiones}

Se obtuvo un sistema que monitorea, desde la Internet, el valor de cinco sensores de CO, instalados en un hospital, a través de una LPWAN y una interfaz de usuario. La LPWAN implantada es fácil de instalar, usar y mantener. El utilizar una solución propietaria, como Symphony Link, permitió realizar de forma rápida una LPWAN eficiente y confiable, disminuyendo el costo de puesta en marcha. La LPWAN puede replicarse en otros ambientes y otro tipo de instalaciones donde sea necesario contar con una solución de IoT para el monitoreo de variables de ambiente. La red es escalable y el diseño de los nodos de la misma permite sustituir el sensor, o adicionar otros, realizando cambios mínimos en la programación del microcontrolador. Si es necesario aumentar el alcance de la LPWAN, puede adicionarse a la misma un transceptor LL-RXR-27 configurado como repetidor. Se dispone del servidor de red y soporte de Link Labs para desarrollos adicionales. Es una solución de monitoreo remoto adecuada para Latinoamérica donde no existen operadores de LPWAN públicas y la opción más viable para conectar a Internet una red LoRa es a través de un gateway.

\section{Referencias}

[1] M. Sezdi and Y. Üzcan, "Clean room classification in the operating room," in 2016 Medical Technologies National Congress (TIPTEKNO), Oct 2016, pp. 1-4. [Online]. Available: https: //doi.org/10.1109/TIPTEKNO.2016.7863107

[2] S. Yalvarmis, A. Ak, and Y. Ülgen, "Which particule sizes and which nutrient medias should be used in order to observe microorganisms in the operating rooms?" in 2014 18th National Biomedical Engineering Meeting, Oct 2014, pp. 1-4. [Online]. Available: https: //doi.org/10.1109/BIYOMUT.2014.7026358

[3] G. Marques and R. Pitarma, "Health informatics for indoor air quality monitoring," in 2016 11th Iberian Conference on Information Systems and Technologies (CISTI), June 2016, pp. 1-6. [Online]. Available: https://doi.org/10.1109/CISTI.2016.7521375 
[4] U. Jaimini, T. Banerjee, W. Romine, K. Thirunarayan, A. Sheth, and M. Kalra, "Investigation of an indoor air quality sensor for asthma management in children," IEEE Sensors Letters, vol. 1, no. 2, pp. 1-4, April 2017. [Online]. Available: https://doi.org/10.1109/LSENS.2017.2691677

[5] S. Sridharan and S. Mangalam, "Carbon monoxide risks and implications on maintenance-intensive fuel-burning appliances - A regulatory perspective," in 2017 Annual Reliability and Maintainability Symposium (RAMS), Jan 2017, pp. 1-7. [Online]. Available: https://doi.org/10.1109/RAM.2017.7889726

[6] Z. Tang, W. Xiong, L. Chen, and N. Jing, "A real-time system for air quality monitoring based on main-memory database," in 201624 th International Conference on Geoinformatics, Aug 2016, pp. 1-4. [Online]. Available: https://doi. org/10.1109/GEOINFORMATICS.2016.7578961

[7] R. M. Liaqat, A. Athar, and N. A. Saqib, "Intelligent agent based system for monitoring and control of hospital management system," in Proceedings of the 2015 2Nd International Conference on Information Science and Security (ICISS), ser. ICISS '15. Washington, DC, USA: IEEE Computer Society, 2015, pp. 1-5. [Online]. Available: http: //dx.doi.org/10.1109/ICISSEC.2015.7371009

[8] E. Lackner, J. Krainer, R. Wimmer-Teubenbacher, F. Sosada, M. Deluca, A. Koeck, E. Laubender, O. Yurchenko, G. Urban, J. Bekacz, K. Rohracher, and E. Wachmann, "Cmos integrated tin dioxide gas sensors functionalized with bimetallic nanoparticles for improved carbon monoxide detection," in 2016 IEEE SENSORS, Oct 2016, pp. 1-3. [Online]. Available: https://doi.org/10.1109/ICSENS.2016.7808667

[9] S. O. Podgornyi, I. P. Demeshko, O. T. Podgornaya, O. V. Lukoyanova, E. D. Skutin, and K. I. Fedotova, "Cadmium telluride nanofilms application in carbon monoxide detection," in 2016 Dynamics of Systems, Mechanisms and Machines (Dynamics), Nov 2016, pp. 1-4. [Online]. Available: https://doi.org/10.1109/Dynamics.2016.7819060

[10] J.-C. Kim, T.-V. Dinh, I.-Y. Choi, and K.-Y. Song, "Physical and chemical factors influencing the continuous monitoring of carbon monoxide using ndir sensor," in 2015 9th International Conference on Sensing Technology (ICST), Dec 2015, pp. 316-319. [Online]. Available: https://doi.org/10.1109/ICSensT.2015.7438414
[11] P. Stefanski, R. Lewicki, J. Tarka, Y. Ma, M. Jahjah, and F. K. Tittel, "Sensitive detection of carbon monoxide using a compact high power cw dfb-qcl based qepas sensor," in CLEO: 2013. Optical Society of America, 2013, pp. 1-2. [Online]. Available: https: //doi.org/10.1364/CLEO_AT.2013.JW2A.68

[12] M. Dong, L. Dianjun, G. Guohui, and X. Jian, "Research on self-calibration carbon monoxide sensor for iron mines," in 2015 12th IEEE International Conference on Electronic Measurement Instruments (ICEMI), vol. 03, July 2015, pp. 1426-1432. [Online]. Available: https://doi.org/10.1109/ICEMI.2015.7494415

[13] J. B. Forsyth, T. L. Martin, D. Young-Corbett, and E. Dorsa, "Feasibility of intelligent monitoring of construction workers for carbon monoxide poisoning," IEEE Transactions on Automation Science and Engineering, vol. 9, no. 3, pp. 505-515, July 2012. [Online]. Available: https://doi.org/10.1109/TASE.2012.2197390

[14] H. Yuan, J. Liu, L. Lei, and H. Han, "Characterizing pollution weather patterns using satellite carbon monoxide data," in 2016 IEEE International Geoscience and Remote Sensing Symposium (IGARSS), July 2016, pp. 5724-5726. [Online]. Available: https://doi.org/10.1109/IGARSS.2016.7730495

[15] J. Liu, K. Ding, and A. Ding, "Vertical sensitivity of satellite remote sensing of atmospheric carbon monoxide," in 2015 IEEE International Geoscience and Remote Sensing Symposium (IGARSS), July 2015, pp. 2234-2237. [Online]. Available: https://doi.org/10.1109/IGARSS.2015.7326250

[16] A. Huong and X. Ngu, "Noninvasive diagnosis of carbon monoxide poisoning using extended modified lambert beer model," in 2014 2nd International Conference on Electronic Design (ICED), Aug 2014, pp. 265-269. [Online]. Available: https://doi.org/10.1109/ICED.2014.7015811

[17] Firdaus, N. Ahriman, A. Yulianto, and M. Kusriyanto, "Wireless sensor network application for carbon monoxide monitoring," in 2015 9th International Conference on Telecommunication Systems Services and Applications (TSSA), Nov 2015, pp. 1-4. [Online]. Available: https://doi.org/10.1109/TSSA.2015.7440434

[18] M. Pies, R. Hajovsky, and S. Ozana, "Wireless measurement of carbon monoxide concentration," in 2014 14th International Conference on Control, Automation and Systems (ICCAS 
2014), Oct 2014, pp. 567-571. [Online]. Available: https://doi.org/10.1109/ICCAS.2014.6987843

[19] S. Son, K. J. Park, and E.-C. Park, "Design of adaptive IEEE 802.11 wlan in hospital environments," in 2013 IEEE 15th International Conference on e-Health Networking, Applications and Services (Healthcom 2013), Oct 2013, pp. 722-724. [Online]. Available: https: //doi.org/10.1109/HealthCom.2013.6720771

[20] M. U. H. A. Rasyid, I. U. Nadhori, and Y. T. Alnovinda, " $\mathrm{CO}$ and $\mathrm{CO}_{2}$ pollution monitoring based on wireless sensor network," in 2015 IEEE International Conference on Aerospace Electronics and Remote Sensing Technology (ICARES), Dec 2015, pp. 1-5. [Online]. Available: https://doi.org/10.1109/ICARES.2015.7429818

[21] H. Ali, J. K. Soe, and S. R. Weller, "A real-time ambient air quality monitoring wireless sensor network for schools in smart cities," in 2015 IEEE First International Smart Cities Conference (ISC2), Oct 2015, pp. 1-6. [Online]. Available: https://doi.org/10.1109/ISC2.2015.7366163

[22] H. A. H. Nograles, C. P. D. Agbay, I. S. L. Flores, A. L. Manuel, and J. B. C. Salonga, "Low cost internet based wireless sensor network for air pollution monitoring using zigbee module," in 2014 Fourth International Conference on Digital Information and Communication Technology and its Applications (DICTAP), May 2014, pp. 310-314. [Online]. Available: https://doi.org/10.1109/DICTAP.2014.6821702

[23] P. A. L. Besari, M. Abdurohman, and A. Rakhmatsyah, "Application of $\mathrm{m} 2 \mathrm{~m}$ to detect the air pollution," in 2015 3rd International Conference on Information and Communication Technology (ICoICT), May 2015, pp. 304-309. [Online]. Available: https://doi.org/10.1109/ICoICT.2015.7231441

[24] H. Jafari, X. Li, L. Qian, and Y. Chen, "Community based sensing: A test bed for environment air quality monitoring using smartphone paired sensors," in 2015 36th IEEE Sarnoff Symposium, Sept 2015, pp. 12-17. [Online]. Available: https://doi.org/10.1109/SARNOF.2015.7324635

[25] A. Bhatta and A. K. Mishra, "Gsm-based commsense system to measure and estimate environmental changes," IEEE Aerospace and Electronic Systems Magazine, vol. 32, no. 2, pp. 54-67, February 2017. [Online]. Available: https://doi.org/10.1109/MAES.2017.150272
[26] M. Werme, T. Eriksson, and T. Righard, "Maintenance concept optimization - A new approach to LORA," in 2017 Annual Reliability and Maintainability Symposium (RAMS), Jan 2017, pp. 1-6. [Online]. Available: https://doi.org/10.1109/RAM.2017.7889740

[27] V. A. Stan, R. S. Timnea, and R. A. Gheorghiu, "Overview of high reliable radio data infrastructures for public automation applications: Lora networks," in 2016 8th International Conference on Electronics, Computers and Artificial Intelligence (ECAI), June 2016, pp. 1-4. [Online]. Available: https://doi.org/10.1109/ECAI.2016.7861130

[28] A. J. Wixted, P. Kinnaird, H. Larijani, A. Tait, A. Ahmadinia, and N. Strachan, "Evaluation of lora and lorawan for wireless sensor networks," in 2016 IEEE SENSORS, Oct 2016, pp. 1-3. [Online]. Available: https://doi.org/10.1109/ICSENS.2016.7808712

[29] L. Gregora, L. Vojtech, and M. Neruda, "Indoor signal propagation of lora technology," in 2016 17th International Conference on Mechatronics Mechatronika (ME), Dec 2016, pp. 1-4. [Online]. Available: https://goo.gl/SFGfYJ

[30] D. Bankov, E. Khorov, and A. Lyakhov, "On the limits of lorawan channel access," in 2016 International Conference on Engineering and Telecommunication (EnT), Nov 2016, pp. 10-14. [Online]. Available: https://doi.org/10.1109/EnT.2016.011

[31] F. Orfei, C. B. Mezzetti, and F. Cottone, "Vibrations powered lora sensor: An electromechanical energy harvester working on a real bridge," in 2016 IEEE SENSORS, Oct 2016, pp. 1-3. [Online]. Available: https://doi.org/10.1109/ICSENS.2016.7808752

[32] L. Li, J. Ren, and Q. Zhu, "On the application of lora lpwan technology in sailing monitoring system," in 2017 13th Annual Conference on Wireless On-demand Network Systems and Services (WONS), Feb 2017, pp. 77-80. [Online]. Available: https://doi.org/10.1109/WONS.2017.7888762

[33] S. Nambiar, A. Nikolaev, M. Greene, L. Cavuoto, and A. Bisantz, "Low-cost sensor system design for in-home physical activity tracking," IEEE Journal of Translational Engineering in Health and Medicine, vol. 4, pp. 1-6, 2016. [Online]. Available: https://doi.org/10.1109/JTEHM.2016.2620971 